\title{
Cerium(III) Chloride: A Highly Efficient Reagent for the Synthesis of $\alpha$-Aminonitriles
}

\section{A. Pasha, H. M. Nanjundaswamy \& V. P. Jayashankara}

To cite this article: M. A. Pasha , H. M. Nanjundaswamy \& V. P. Jayashankara (2007)

Cerium(III) Chloride: A Highly Efficient Reagent for the Synthesis of $\alpha \#$ Aminonitriles, Synthetic Communications, 37:24, 4371-4380, DOI: 10.1080/00397910701578180

To link to this article: http://dx.doi.org/10.1080/00397910701578180

曲 Published online: 14 Dec 2007.

Submit your article to this journal $\widetilde{ }$

山 Article views: 66

Q View related articles $\sqsubset$

Citing articles: 11 View citing articles 지 


\title{
Cerium(III) Chloride: A Highly Efficient Reagent for the Synthesis of $\alpha$-Aminonitriles
}

\author{
M. A. Pasha \\ Department of Studies in Chemistry, Bangalore University, Bangalore, \\ India \\ H. M. Nanjundaswamy \\ Chemical Examination Section, Public Health Institute, Bangalore, India \\ V. P. Jayashankara \\ Department of Studies in Chemistry, Bangalore University, Bangalore, \\ India
}

\begin{abstract}
We report a highly efficient, one-pot, three-component condensation of carbonyl compounds, amines, and TMSCN in MeCN; the reaction is significantly promoted by the catalytic amount of cerium(III) chloride at ambient temperature in excellent yields without any adverse effect on the other substituents. The method afforded an elegant alternative to the synthesis of $\alpha$-aminonitriles. The reactions are fast and clean, and the products obtained are of high purity.
\end{abstract}

Keywords: amines, $\alpha$-aminonitriles, carbonyl compounds, cerium(III) chloride, one-pot three-component condensation

\section{INTRODUCTION}

$\alpha$-Aminonitriles are useful intermediates in the synthesis of amino acids and nitrogen-containing heterocycles. ${ }^{[1]}$ Among many other applications, they are readily converted to diamines, which are of interest as ligands for platinum(II)

Received in India May 21, 2007

Address correspondence to M. A. Pasha, Department of Studies in Chemistry, Bangalore University, Central College Campus, Bangalore 560 001, India. E-mail: m_af_pasha@yahoo.co.in 
complexes with potential antitumor properties. ${ }^{[2]}$ Although several procedures have been devised for the synthesis of $\alpha$-aminonitriles, the Strecker reaction provides one of the most efficient methods. The classical Strecker reaction is generally carried out with alkali cyanides in aqueous solution, which has some limitations; consequently several modifications of the Strecker reaction have been reported using a variety of cyanating agents such as tributyltin cyanide $\left(\mathrm{Bu}_{3} \mathrm{SnCN}\right), \alpha$-trimethylsiloxynitriles, and diethyl phosphorocyanides under various reaction conditions. ${ }^{[3]}$ Trimethylsilylcyanide (TMSCN) is a cyanide ion source that provides a promising and safer route to this class of reactions. ${ }^{[4]}$

Recently, one-pot procedures for the synthesis of $\alpha$-aminonitriles from carbonyl compounds have been developed in the presence of a variety of catalysts such as $\mathrm{InCl}_{3},{ }^{[5]} \mathrm{BiCl}_{3},{ }^{[6]} \mathrm{NiCl}_{2},{ }^{[7]} \mathrm{CoCl}_{2},{ }^{[8]} \mathrm{Sc}(\mathrm{OTf})_{3},{ }^{[9]} \mathrm{Cd}(\mathrm{II})$ salt, ${ }^{[10]} \mathrm{Pt}$ salt, ${ }^{[11]} \mathrm{I}_{2},{ }^{[12]}$ and montmorillonite KSF clay. ${ }^{[13]}$

\section{RESULTS AND DISCUSSION}

In continuation of our research program on organic transformations employing easily available and inexpensive reagents under mild conditions at short durations, recently we have synthesized some medicinally important molecules. ${ }^{[14]}$ Because of the significance of $\alpha$-aminonitriles, herein we report a clean procedure for the synthesis of a variety of $\alpha$-aminonitriles under mild conditions using catalytic cerium(III) chloride. The reaction is efficient and proceeds at room temperature either by the addition of a primary or secondary amine to a mixture of aldehyde/trimethylsilyl cyanide (TMSCN) or by the addition of an aldehyde to a mixture of amine/TMSCN in acetonitrile in the presence of a catalytic amount of cerium chloride to give a high yield of the product as shown in Scheme 1.

We chose benzaldehyde, aniline, and TMSCN as a representative reaction performed in the presence of catalytic $\mathrm{CeCl}_{3}$ in various organic solvents (viz., methanol, dichloromethane, dichloroethane, tetrahydrofuran, ethanol, and acetonitrile). The reaction proceeds smoothly at room temperature, giving the corresponding $\alpha$-aminonitrile in high yield (95\%). Among the solvents examined, acetonitrile emerged as superior medium that gave excellent results. The condensation of different aldehydes and amines was performed under these conditions to get $\alpha$-aminonitriles within 1.25 to $1.5 \mathrm{~h}$ (except entry 16). The course of reaction was monitored by thin-layer chromatography (TLC). All the products obtained by this system (listed in Table 1) were characterized by their melting

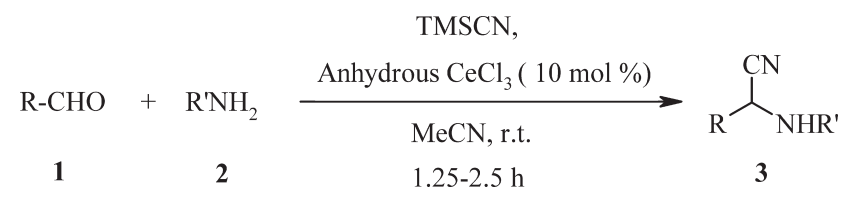

Scheme 1. 
Table 1. Facile preparation of $\alpha$-aminonitriles catalyzed by cerium chloride

\begin{tabular}{|c|c|c|c|c|c|}
\hline Entry & 1 & 2 & $3^{a}$ & $\begin{array}{l}\text { Time } \\
\text { (h) }\end{array}$ & $\begin{array}{l}\text { Yield } \\
(\%)^{b}\end{array}$ \\
\hline 1 & $\mathrm{C}_{6} \mathrm{H}_{5} \mathrm{CHO}$ & $\mathrm{C}_{6} \mathrm{H}_{5} \mathrm{NH}_{2}$ & & 1.25 & 95 \\
\hline 2 & $\mathrm{C}_{6} \mathrm{H}_{5} \mathrm{CHO}$ & $\mathrm{C}_{6} \mathrm{H}_{5} \mathrm{CH}_{2} \mathrm{NH}_{2}$ & & 1.50 & 92 \\
\hline 3 & $\mathrm{C}_{6} \mathrm{H}_{5} \mathrm{CHO}$ & 2- $\mathrm{MeC}_{6} \mathrm{H}_{4} \mathrm{NH}_{2}$ & & 1.25 & 94 \\
\hline 4 & $4-\mathrm{OMeC}_{6} \mathrm{H}_{4} \mathrm{CHO}$ & $\mathrm{C}_{6} \mathrm{H}_{5} \mathrm{NH}_{2}$ & & 1.25 & 96 \\
\hline 5 & $4-\mathrm{OMeC}_{6} \mathrm{H}_{4} \mathrm{CHO}$ & 2- $\mathrm{MeC}_{6} \mathrm{H}_{4} \mathrm{NH}_{2}$ & & 1.25 & 90 \\
\hline 6 & 2-OEtC ${ }_{6} \mathrm{H}_{4} \mathrm{CHO}$ & $\mathrm{C}_{6} \mathrm{H}_{5} \mathrm{NH}_{2}$ & & 1.25 & 94 \\
\hline 7 & $2-\mathrm{OEtC}_{6} \mathrm{H}_{4} \mathrm{CHO}$ & $2-\mathrm{MeC}_{6} \mathrm{H}_{4} \mathrm{NH}_{2}$ & & 1.25 & 90 \\
\hline
\end{tabular}


Table 1. Continued

\begin{tabular}{|c|c|c|c|c|c|}
\hline Entry & 1 & 2 & $3^{a}$ & $\begin{array}{l}\text { Time } \\
\text { (h) }\end{array}$ & $\begin{array}{l}\text { Yield } \\
(\%)^{b}\end{array}$ \\
\hline 8 & $2-\mathrm{OEtC}_{6} \mathrm{H}_{4} \mathrm{CHO}$ & $\mathrm{C}_{6} \mathrm{H}_{5} \mathrm{C}_{2} \mathrm{H}_{4} \mathrm{NH}_{2}$ & & 1.25 & 68 \\
\hline 9 & 4- $\mathrm{ClC}_{6} \mathrm{H}_{4} \mathrm{CHO}$ & $\mathrm{C}_{6} \mathrm{H}_{5} \mathrm{NH}_{2}$ & & 1.25 & 91 \\
\hline 10 & $4-\mathrm{MeC}_{6} \mathrm{H}_{4} \mathrm{CHO}$ & $\mathrm{C}_{6} \mathrm{H}_{5} \mathrm{NH}_{2}$ & & 1.25 & 90 \\
\hline 11 & $\mathrm{C}_{6} \mathrm{H}_{5} \mathrm{CHO}$ & 4- $\mathrm{ClC}_{6} \mathrm{H}_{4} \mathrm{NH}_{2}$ & & 1.25 & 92 \\
\hline 12 & $\mathrm{C}_{6} \mathrm{H}_{5} \mathrm{CHO}$ & 4- $\mathrm{OMeC}_{6} \mathrm{H}_{4} \mathrm{NH}_{2}$ & & 1.25 & 92 \\
\hline 13 & $\leadsto \mathrm{CHO}$ & $\mathrm{C}_{6} \mathrm{H}_{5} \mathrm{NH}_{2}$ & & 1.75 & 90 \\
\hline 14 & यौ & $\mathrm{C}_{6} \mathrm{H}_{5} \mathrm{NH}_{2}$ & & 1.25 & 91 \\
\hline
\end{tabular}




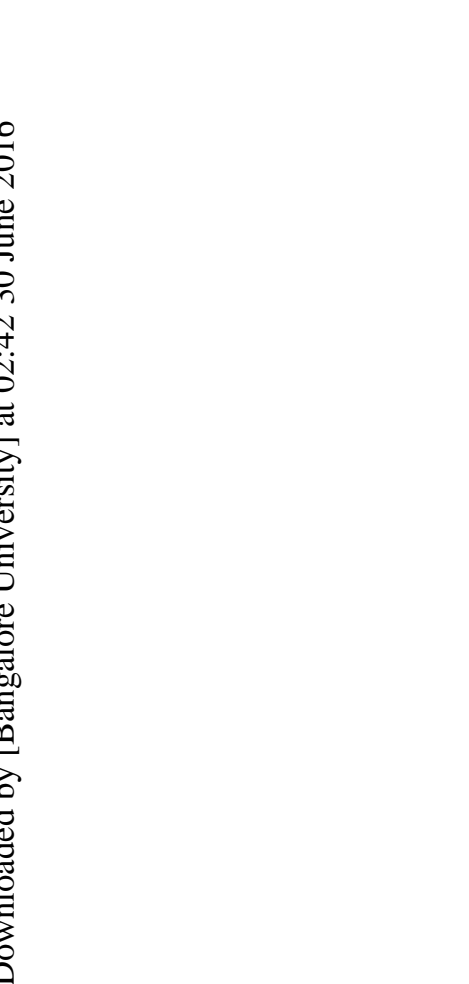

15

17

19

21

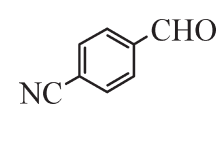

$\mathrm{C}_{6} \mathrm{H}_{5} \mathrm{CHO}$

${ }_{\mathrm{O}}^{\lambda} \mathrm{CHO}$

总

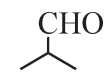

$\sim_{\mathrm{CHO}}$

$\sim_{\mathrm{CHO}}$
$\mathrm{C}_{6} \mathrm{H}_{5} \mathrm{NH}_{2}$

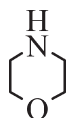

2- $\mathrm{MeC}_{6} \mathrm{H}_{4} \mathrm{NH}_{2}$

$\mathrm{C}_{6} \mathrm{H}_{5} \mathrm{NH}_{2}$

2- $\mathrm{MeC}_{6} \mathrm{H}_{4} \mathrm{NH}_{2}$

$\mathrm{C}_{6} \mathrm{H}_{5} \mathrm{NH}_{2}$

$2-\mathrm{MeC}_{6} \mathrm{H}_{5} \mathrm{NH}_{2}$

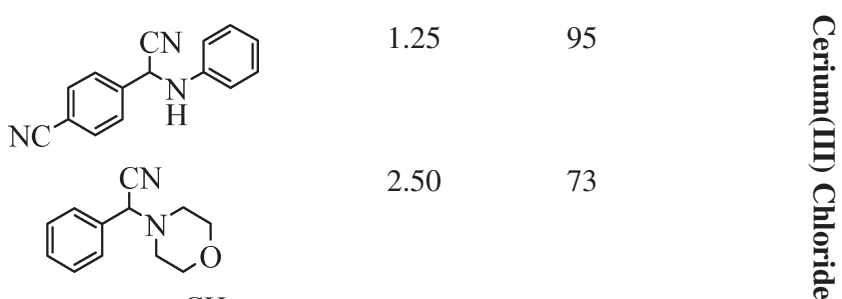

(1)

$\searrow_{\mathrm{CN}}^{\mathrm{H}} \longrightarrow$

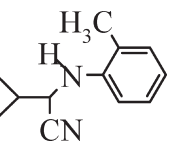

1.25

1.2593

$1.25 \quad 9$ 
Table 1. Continued

\begin{tabular}{lllll}
\hline Entry & 1 & 2 & $\begin{array}{c}\text { Time } \\
(\mathrm{h})\end{array}$ & $\begin{array}{c}\text { Yield } \\
(\%)^{b}\end{array}$ \\
\hline 22 & $\mathrm{C}_{6} \mathrm{H}_{5} \mathrm{CHO}$ & $3^{a}$ & 1.25 \\
\hline
\end{tabular}

${ }^{a}$ All the products are thoroughly characterized by ${ }^{1} \mathrm{H}$ NMR, GC-MS, and IR spectral analysis.

${ }^{b}$ Yields refer to the products after purification by column chromatography. 
points/physical state, TLC, IR, and ${ }^{1} \mathrm{H}$ NMR spectra in comparison with authentic samples.

\section{EXPERIMENTAL}

Cerium chloride, other reagents, and solvents were all commercial samples and were purified as per the requirement. TLC was carried out on silica-gel plates. All reactions were performed under an ambient atmosphere. Melting points were measured on a Büchi B-540 apparatus; IR and ${ }^{1} \mathrm{H}$ NMR spectra were recorded on Nicolet 400D FT-IR and Bruker AMX (200-MHz) spectrometers respectively. Gas chromatographic (GC) analyses were performed on a Shimadzu GC-MS QP 5050A instrument. Yields refer to the isolated products after purification by 60 - to 120-mesh silica-gel column chromatography using 15:85 ethyl acetate-hexane $(\mathrm{v} / \mathrm{v})$ as an eluent.

\section{CONCLUSION}

In conclusion, we have demonstrated a highly efficient, convenient, inexpensive, and facile route for one-pot, three-component condensation of carbonyl compounds, amines, and TMSCN in acetonitrile in the presence of a catalytic amount of cerium chloride without the need for an inert atmosphere. The procedure includes simple workup and shorter reaction periods and affords excellent yields of the product; it is more effective than earlier reports. Most of the reactions were completed in $1.25 \mathrm{~h}$ at ambient temperature; the products were analytically pure. This procedure will be of general use and interest to the synthetic chemistry community.

\section{Typical Procedure for the Preparation of 2-( $N$-Anilino)-2- (phenyl)acetonitrile (3.1)}

A mixture of benzaldehyde (106 mg, $1 \mathrm{mmol})$, aniline (93 mg, $1 \mathrm{mmol})$, and trimethylsilyl cyanide $(129 \mathrm{mg}, 1.3 \mathrm{mmol})$ in acetonitrile $(10 \mathrm{~mL})$ was stirred at room temperature in the presence of $\mathrm{CeCl}_{3}(25 \mathrm{mg}, 10 \mathrm{~mol} \%)$. After completion of the reaction (TLC), the solvent was removed in vacuo, quenched with water $(15 \mathrm{~mL})$, and extracted with ethyl acetate $(3 \times 10 \mathrm{~mL})$. The organic layer was washed with water $(20 \mathrm{~mL})$ and brine $(20 \mathrm{~mL})$, then dried over $\mathrm{MgSO}_{4}$ and concentrated. The residue was chromatographed over silica gel (ethyl acetate-hexane 1.5:8.5) to afford the pure product. 


\section{Spectral Data of Selected Products}

3.1. White crystalline solid. $\mathrm{Mp}$ found, $73-74^{\circ} \mathrm{C}$; reported, $73-74^{\circ} \mathrm{C}$. ${ }^{1} \mathrm{H}$ NMR $\left(\mathrm{CDCl}_{3}\right): \delta(4.0(\mathrm{~d}, 1 \mathrm{H}, J=8.1 \mathrm{~Hz}), 5.40(\mathrm{~d}, 1 \mathrm{H}, J=8.1 \mathrm{~Hz})$, $6.75(\mathrm{~d}, 2 \mathrm{H}, J=8.0 \mathrm{~Hz}), 6.90(\mathrm{t}, 1 \mathrm{H}, J=7.8 \mathrm{~Hz}), 7.25(\mathrm{t}, 2 \mathrm{H}$, $J=7.8 \mathrm{~Hz}), 7.40-7.50(\mathrm{~m}, 3 \mathrm{H}), 7.60-7.70(\mathrm{~m}, 2 \mathrm{H})$. Mass: $\mathrm{m} / \mathrm{z}: 208$ $\mathrm{M}^{+}, 180,116,91,77$.

3.2. Colorless oil. ${ }^{1} \mathrm{H}$ NMR $\left(\mathrm{CDCl}_{3}\right)$ : $\delta(1.80$ (brs, $1 \mathrm{H}, \mathrm{NH}), 3.95(\mathrm{~d}, 2 \mathrm{H}$, $J=13.5 \mathrm{~Hz}), 4.70(\mathrm{~s}, 1 \mathrm{H}), 6.78(\mathrm{~d}, 1 \mathrm{H}, J=8.0 \mathrm{~Hz}), 7.15(\mathrm{t}, 1 \mathrm{H}$, $J=7.8 \mathrm{~Hz}), 7.25-7.40(\mathrm{~m}, 6 \mathrm{H}), 7.49-7.51(\mathrm{~m}, 2 \mathrm{H})$. Mass: $\mathrm{m} / \mathrm{z}: 222 \mathrm{M}^{+}$, $195,141,131,116,106,91,77,51$.

3.3. Pale yellow solid. Mp found, $73-74^{\circ} \mathrm{C}$; reported, $72-73^{\circ} \mathrm{C} .{ }^{1} \mathrm{H}$ NMR $\left(\mathrm{CDCl}_{3}\right): \delta(2.20(\mathrm{~s}, 3 \mathrm{H}), 3.38($ broad, $1 \mathrm{H}, \mathrm{NH}, J=8.1 \mathrm{~Hz}), 5.45(\mathrm{~d}, 1 \mathrm{H}$, $J=8.1 \mathrm{~Hz}), 6.80(\mathrm{t}, 2 \mathrm{H}, J=7.9 \mathrm{~Hz}), 7.10(\mathrm{~d}, 1 \mathrm{H}, J=8.0 \mathrm{~Hz}), 7.20(\mathrm{~d}, 1 \mathrm{H}$, $J=7.9 \mathrm{~Hz}), 7.40-7.50(\mathrm{~m}, 3 \mathrm{H}), 7.50(\mathrm{~d}, 2 \mathrm{H}, J=8.0 \mathrm{~Hz})$. Mass: $\mathrm{m} / \mathrm{z}: 222$ $\mathrm{M}^{+}, 194,155,141,116,106,91,73,65,45$.

3.4. White solid. Mp found, $94-95^{\circ} \mathrm{C}$; reported, $94-95^{\circ} \mathrm{C} .{ }^{1} \mathrm{H}$ NMR $\left(\mathrm{CDCl}_{3}\right)$ : $\delta(3.80(\mathrm{~s}, 3 \mathrm{H}), 3.90(\mathrm{~d}, 1 \mathrm{H}, J=8.1 \mathrm{~Hz}), 5.30(\mathrm{~d}, 1 \mathrm{H}, J=8.1 \mathrm{~Hz}), 6.75(\mathrm{~d}, 2 \mathrm{H}$, $J=8.0 \mathrm{~Hz}), 6.80(\mathrm{t}, 1 \mathrm{H}, J=7.9 \mathrm{~Hz}), 6.90(\mathrm{~d}, 2 \mathrm{H}, J=8.0 \mathrm{~Hz}), 7.25(\mathrm{t}, 2 \mathrm{H}$, $J=7.9 \mathrm{~Hz}), 7.50(\mathrm{~d}, 2 \mathrm{H}, J=8.0 \mathrm{~Hz})$. Mass: $\mathrm{m} / \mathrm{z}: 238 \mathrm{M}^{+}, 211,181,167$, $141,104,77,51,40$.

3.7. Yellow crystal. Mp found, $70-71{ }^{\circ} \mathrm{C}$; reported, $69-72^{\circ} \mathrm{C} .{ }^{1} \mathrm{H}$ NMR $\left(\mathrm{CDCl}_{3}\right): \delta(1.50(\mathrm{t}, 3 \mathrm{H}, J=7.9 \mathrm{~Hz}), 2.13(\mathrm{~s}, 3 \mathrm{H}), 4.13(\mathrm{~m}, 2 \mathrm{H}), 4.21$ (brs, $1 \mathrm{H}), 5.43(\mathrm{~d}, 1 \mathrm{H}, J=8.9 \mathrm{~Hz}), 6.87-6.99(\mathrm{~m}, 4 \mathrm{H}), 7.23-7.45(\mathrm{~m}, 4 \mathrm{H})$. Mass: $\mathrm{m} / \mathrm{z}: 266 \mathrm{M}^{+}$.

3.9. White solid. Mp found, $110-112^{\circ} \mathrm{C}$; reported, $109-112^{\circ} \mathrm{C} .{ }^{1} \mathrm{H}$ NMR $\left(\mathrm{CDCl}_{3}\right): \delta(4.0(\mathrm{~d}, 1 \mathrm{H}, J=8.1 \mathrm{~Hz}), 5.39(\mathrm{~d}, 1 \mathrm{H}, J=8.1 \mathrm{~Hz}), 6.75(\mathrm{~d}, 2 \mathrm{H}$, $J=8.0 \mathrm{~Hz}), 6.90(\mathrm{t}, 1 \mathrm{H}, J=7.9 \mathrm{~Hz}), 7.15(\mathrm{t}, 2 \mathrm{H}, J=7.9 \mathrm{~Hz}), 7.40(\mathrm{~d}, 2 \mathrm{H}$, $J=8.0 \mathrm{~Hz}), 7.60(\mathrm{~d}, 2 \mathrm{H}, J=8.0 \mathrm{~Hz})$. Mass: $\mathrm{m} / \mathrm{z}: 242 \mathrm{M}^{+}, 213,149,114$, $91,73,59$.

3.10. Yellow solid. Mp found, $77-79^{\circ} \mathrm{C}$; reported, $76-78^{\circ} \mathrm{C}$; ${ }^{1} \mathrm{H}$ NMR $\left(\mathrm{CDCl}_{3}\right): \delta(2.40(\mathrm{~s}, 3 \mathrm{H}), 6.78(\mathrm{~d}, 2 \mathrm{H}, J=8.0 \mathrm{~Hz}), 6.90(\mathrm{t}, 1 \mathrm{H}, J=7.8 \mathrm{~Hz})$, $7.20-7.30(\mathrm{~m}, 4 \mathrm{H}), 7.50(\mathrm{~d}, 2 \mathrm{H}, J=8.0 \mathrm{~Hz})$. Mass: m/z: $222 \mathrm{M}^{+}, 194$, 176, 131, 103, 91, 77, 41.

3.11. Pale yellow solid. Mp found, $107-110^{\circ} \mathrm{C}$; reported, $108-110^{\circ} \mathrm{C} .{ }^{1} \mathrm{H}$ $\operatorname{NMR}\left(\mathrm{CDCl}_{3}\right): \delta(4.07(\mathrm{~d}, 4 \mathrm{H}, J=8.0 \mathrm{~Hz}), 5.29(\mathrm{~d}, 1 \mathrm{H}, J=8.0 \mathrm{~Hz}), 6.61$ $(\mathrm{d}, 2 \mathrm{H}, J=8.0 \mathrm{~Hz}), 7.51(\mathrm{~d}, 2 \mathrm{H}, J=8.0 \mathrm{~Hz}), 7.47-7.34(\mathrm{~m}, 3 \mathrm{H}), 7.60-$ 7.48 (m, 2H). Mass: m/z: $242 \mathrm{M}^{+}, 215,178,126$. 
3.12. Pale yellow solid. Mp found, $95-96^{\circ} \mathrm{C}$; reported: $94-96^{\circ} \mathrm{C} .{ }^{1} \mathrm{H}$ NMR $\left(\mathrm{CDCl}_{3}\right): \delta(3.72(\mathrm{~s}, 3 \mathrm{H}) .3 .84(\mathrm{~d}, 1 \mathrm{H}, J=8.0 \mathrm{~Hz}), 5.29(\mathrm{~d}, 1 \mathrm{H}$, $J=8.0 \mathrm{~Hz}), 6.67(\mathrm{~d}, 2 \mathrm{H}, J=8.0 \mathrm{~Hz}), 6.78(\mathrm{~d}, 2 \mathrm{H}, J=8.0 \mathrm{~Hz}), 7.47-7.36$ (m, 3H), 7.62-7.51 (m, 2H). Mass: m/z: $238 \mathrm{M}^{+}, 211,210,146$.

3.13. Pale yellow solid. $\mathrm{Mp}$ found, $118-120^{\circ} \mathrm{C}$; reported: $117-119^{\circ} \mathrm{C}$. ${ }^{1} \mathrm{H}$ NMR $\left(\mathrm{CDCl}_{3}\right): \delta(3.80(\mathrm{~d}, 1 \mathrm{H}, J=8.1 \mathrm{~Hz}), 5.05(\mathrm{~m}, 1 \mathrm{H}), 6.30(\mathrm{dd}, 1 \mathrm{H}$, $J=6.9,17.3 \mathrm{~Hz}), 6.78(\mathrm{~d}, 1 \mathrm{H}, J=8.0 \mathrm{~Hz}), 6.90(\mathrm{t}, 1 \mathrm{H}, J=7.9 \mathrm{~Hz}), 7.08$ $(\mathrm{dd}, 1 \mathrm{H}, J=1.7,17.3 \mathrm{~Hz}), 7.25-7.45(\mathrm{~m}, 8 \mathrm{H})$. Mass: $\mathrm{m} / \mathrm{z}: 234 \mathrm{M}^{+}, 206$, $128,115,77,51$.

3.14. Dark brown solid. Mp found, $68-69^{\circ} \mathrm{C}$; reported: $68-70{ }^{\circ} \mathrm{C} .{ }^{1} \mathrm{H}$ NMR $\left(\mathrm{CDCl}_{3}\right): \delta(4.05(\mathrm{~d}, 1 \mathrm{H}, J=8.1 \mathrm{~Hz}), 5.40(\mathrm{~d}, 1 \mathrm{H}, J=8.1 \mathrm{~Hz}), 6.40$ $(\mathrm{m}, 1 \mathrm{H}), 6.55(\mathrm{~m}, 1 \mathrm{H}), 6.80(\mathrm{~d}, 2 \mathrm{H}, J=8.0 \mathrm{~Hz}), 6.90(\mathrm{t}, 1 \mathrm{H}, J=7.9 \mathrm{~Hz})$, $7.25(\mathrm{t}, 2 \mathrm{H}, J=7.9 \mathrm{~Hz}), 7.40(\mathrm{~m}, 1 \mathrm{H})$. Mass: $\mathrm{m} / \mathrm{z}: 198 \mathrm{M}^{+}, 169,155,141$, $115,106,92,77,51$.

3.15. Pale yellow solid. Mp $112-114^{\circ} \mathrm{C} .{ }^{1} \mathrm{H}$ NMR $\left(400 \mathrm{MHz}, \mathrm{CDCl}_{3}\right): \delta(5.57$ $(\mathrm{s}, 1 \mathrm{H}, \mathrm{NH}), 6.79-6.81(\mathrm{~d}, 2 \mathrm{H}, J=8.1 \mathrm{~Hz}), 6.99(\mathrm{~s}, 1 \mathrm{H}), 7.26(\mathrm{~d}, 2 \mathrm{H}), 7.73$ (s, 5H). Mass: m/z: $234 \mathrm{M}^{+}, 207,146$.

3.20. Pale yellow oil, ${ }^{1} \mathrm{H}$ NMR $\left(\mathrm{CDCl}_{3}\right): \delta(0.95(\mathrm{t}, 3 \mathrm{H}, J=7.0 \mathrm{~Hz}), 1.20-$ $1.56(\mathrm{~m}, 6 \mathrm{H}), 1.84-1.92(\mathrm{~m}, 2 \mathrm{H}), 4.10-4.18(\mathrm{~m}, 2 \mathrm{H}), 6.65(\mathrm{~d}, 2 \mathrm{H}$, $J=8.0 \mathrm{~Hz},), 6.85(\mathrm{t}, 1 \mathrm{H}, J=8.0 \mathrm{~Hz}) 7.25(\mathrm{t}, 2 \mathrm{H}, J=8.0 \mathrm{~Hz})$. Mass: $\mathrm{m} / \mathrm{z}$ : $202 \mathrm{M}^{+}, 187,175,146$.

\section{REFERENCES}

1. (a) Weinstock, L. M.; Davis, P.; Handelsman, B.; Tull, R. J. Org. Chem. 1967, 32, 2823-2829; (b) Matier, W. L.; Owens, D. A.; Comer, W. T.; Deitchman, D.; Ferguson, H. C.; Seidehamel, R. J.; Young, J. R. J. Med. Chem. 1973, 16, 901-908; (c) Shafran, Y. M.; Bakulev, V. A.; Mokrushin, V. S. Russ. Chem. Rev. 1989, 58, 148-162.

2. (a) Brunner, H.; Hankofer, P.; Holzinger, U.; Treittinger, B.; Schonenberger, H. Eur. J. Med. Chem. 1990, 25, 35-44; (b) Marco, J. L.; Ingate, S. T.; Manzano, P. Tetrahedron Lett. 1998, 39, 4123-4124; (c) Marco, J. L.; Ingate, S. T. Tetrahedron Lett. 1997, 38, 4835-4836.

3. (a) Mai, K.; Patil, G. Tetrahedron Lett. 1984, 25, 4583-4586; (b) Mai, K.; Patil, G. Synth. Commun. 1985, 15, 157-163; (c) Mori, M.; Imma, H.; Nakai, T. Tetrahedron Lett. 1997, 38, 6229-6232; (d) Mulzer, J.; Meier, A.; Buschmam, J.; Luger, P. Synthesis 1996, 123-132.

4. Ranu, B. C.; Dey, S. S.; Hajra, A. Tetrahedron 2002, 58, 2529-2532.

5. (a) Heydari, A.; Fatemi, P.; Alizadey, A. A. Tetrahedron Lett. 1998, 39, 3409-3412; (b) Chakraborthy, T. K.; Reddy, G. V.; Hussein, K. A. Tetrahedron Lett. 1991, 32, 7597-7600.

6. De, S. K.; Gibbs, R. A. Tetrahedron Lett. 2004, 45, 7407-7408. 
7. De, S. K. J. Mol. Catal. A 2005, 225, 169-171.

8. De, S. K. Beilstein J. Org. Chem. 2005, 1, 8.

9. Kobayashi, S.; Basujima, T.; Nagayama, S. J. Chem. Soc., Chem. Commun. 1998, 981-982.

10. Ohmuri, O.; Fujita, M. J. Chem. Soc., Chem. Commun. 2004, 1586-1587.

11. Fossey, J. S.; Richards, C. J. Tetrahedron Lett. 2003, 44, 8773-8776.

12. Royer, L.; De, S. K.; Gibbs, R. A. Tetrahedron Lett. 2005, 46, 4595-4597.

13. Yadav, J. S.; Reddy, B. V.; Eeshwaraih, B.; Srinivas, M. Tetrahedron 2004, 60 , 1767-1771.

14. (a) Pasha, M. A.; Jayashankara, V. P. Heterocycles 2006, 68, 1017-1023; (b) Pasha, M. A.; Jayashankara, V. P. Bio. Org. Med. Chem. Lett. 2007, 17, 621-623. 